\title{
Recurrent Rhinosporidiosis
}

\author{
Indira Acharya*, Ashik Pokharel, Ashbita Pokharel and Bibhu Pradhan \\ GMSMA of ENT-HN Studies, MMC-TUTH, IOM, Nepal
}

Submission: August 15, 2017; Published: August 24, 2017

*Corresponding author: Indira Acharya, GMSMA of ENT-HN Studies, MMC-TUTH, IOM, Nepal, Tel: 977-9849485822;

Email: acharyaindira4@gmail.com

\begin{abstract}
Rhinosporidiosis is a rare, chronic granulomatous disease, caused by aquatic protistan parasite Rhinosporidium seeberi [1]. It affects predominantly mucous membrane of nose and nasopharynx but occasionally lips, palate, uvula, maxillary antrum, conjunctiva, lacrimal sac, epiglottis, larynx, trachea, bronchus, scalp, penis, vulva \& vagina. In Nepal it occurs in Terai belt mainly Janakpur, Sarlahi \& Rajbiraj districts. We report a case of recurrent rhinosporidiosis affecting nasal cavity, nasopharynx \& oropharynx. Patient is 31yrs old female from Janakpur who previously underwent surgical excision twice. She now presents recurrent, flesh like lesion at previous excision sites. This time surgical excision with base cauterization was done. Wide local excision with electro cauterization is the treatment of choice with postoperative systematic Dapsone that may be prescribed for up to 1 year. However, local recurrence remains a problem, likely secondary to auto-inoculation during the surgical trauma.
\end{abstract}

\section{Introduction}

Rhinosporidiosis is a rare chronic granulomatous disease, caused by the parasite Rhinosporidium seeberi [1-3]. The disease is commonly found in the anterior nares, the inferior turbinate, septum and floor of the nasal cavity; however, it can also affect extranasal sites including the oral cavity, trachea and uvula [1,4-11]. Infection is usually limited to surface epithelium but sometimes there may be wide dissemination with visceral involvement [11]. It is usually a result of direct traumatic inoculation with the organism. Trauma from surgery has also been cited as potential mode of transmission $[1,7,9,10]$. The disease progresses with local replication of R. seeberi and results in hyperplastic growth of host tissue and associated local immune response [1,2]. The disease is prevalent mainly in Nepal, Indian subcontinent [1,8], and Srilanka. Definitive guidelines regarding surgical and medical treatment are poorly covered in the literature. Although rare in other countries, immigration from areas of the world where it exists increases the likelihood of incidence of the disease [3]. We report a case of recurrent rhinosporidiosis in a Neplease female affecting nasal cavity, nasopharynx \& oropharynx, who presented with globus pharyngeus and dysphagia. Surgical excision \& base cauterization was done.

\section{Case Report}

We present a case report of a 31yrs old female from Janakpur, Nepal, presented to Bir Hospital, 11 years ago with complain of dysphagia and feeling of lump in her throat. Her symptoms were progressive. Nasopharyngolaryngoscopy showed nasopharyngeal soft tissue mass. She underwent surgical excision \& was on regular follow up. Seven years later she again presented to Bir Hospital with similar complaint. Nasopharyngolaryngoscopy showed signs of recurrent lesion in nasopharynx and left nasal cavity. She underwent excision of mass under General anesthesia \& was better. She now presents with complains of lump in throat from past $4 \mathrm{yrs}$. She also had complains of bilateral nasal obstruction from past 3 months. Nasal obstruction was insidious in onset, gradually progressive complete and associated with pain over dorsum of nose, hyponasal voice, intermittent bleeding from bilateral nasal cavities, mouth breathing and excessive nasal discharge.

On examination, a flesh-like, reddish, exophytic and lobular mass was seen to occupy oropharynx (Figure 1). It was nonpulsating or expansile \& did not bleed on touch. The mass had a pedicle attached to the nasopharynx, which suggested it to be its origin. The rest of his oral cavity was unremarkable. Anterior rhinoscopy showed bilateral pinkish polypoidal mass filling both the nasal cavities. The nasal mucosa was swollen, hyperemic, and covered with copious viscid secretion. On patency test, there was B/L decreased air entry. There was no cervical lymphadenopathy. Nasopharyngolaryngoscopy was performed which confirmed the origin of the lesion from uvula, soft palate \& nasopharynx. Further evaluation with a CT scan demonstrated soft tissue density in Right nasal cavity (Figure 2). 


\section{Global Journal of Otolaryngology}

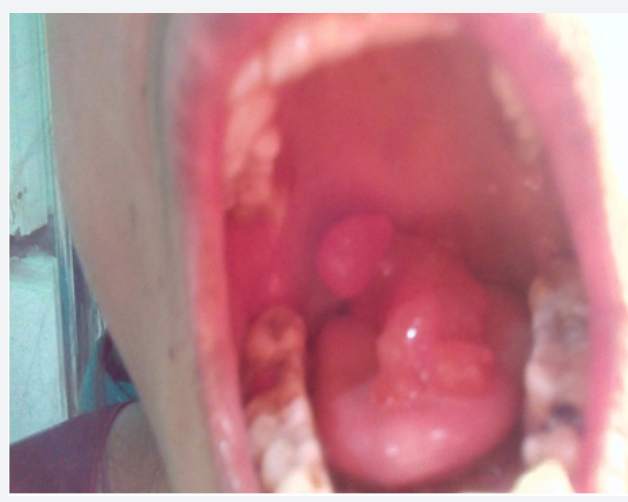

Figure 1: Red pinkish irregular mass in oropharynx.

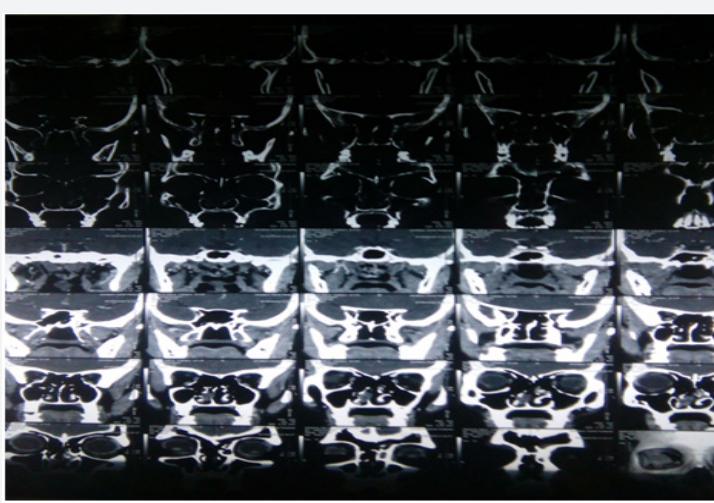

Figure 2: CT nose \& paranasal sinuses coronal view.

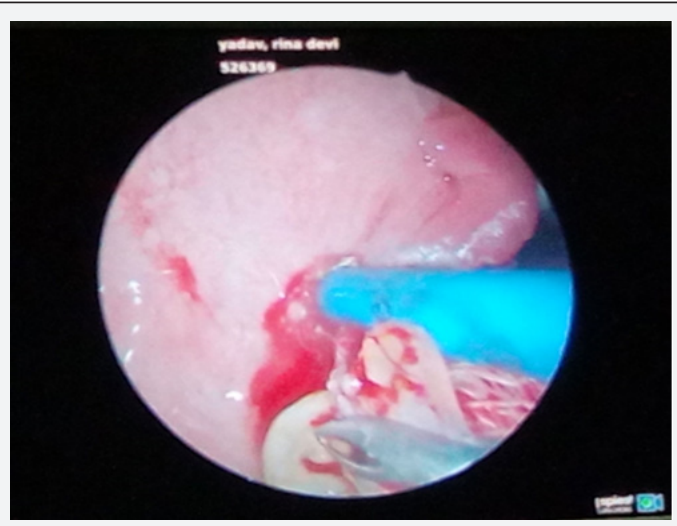

Figure 3 : Tranoral excision of mass using monopolar suction cautery.

She was planned for surgical excision. Under General anesthesia, patient was placed in supine in Reverse trendelenberg position. Root of the tumor in the nasopharynx was exposed by placing Boyles Davis mouth gag with tongue blade. Nasopharyngeal mass was excised transorally using monopolar suction cautery (Figure 3). Zero degree rigid endoscope was inserted through nasal cavity to assess pathology of mass. Polypoidal mass in bilateral nasal cavities were excised and sent for histopathological examination. Attachment sites of mass cauterized with monopolar cautery. Bilateral posterior nasal packing placed. Per operative finding showed pale polypoidal mass along with some pinkish masses with irregular surface and few sporangia on them (Figure 4) with multiple attachments:

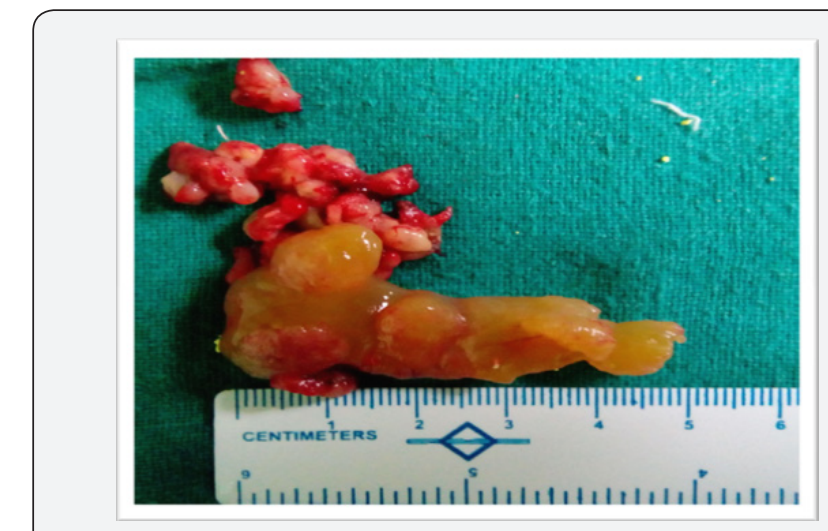

Figure 4 : Per operative finding showing pale polypoidal mass along with some pinkish masses.
a) Posterior surface of uvula
b) Superior surface of soft palate
c) Poster superior wall of nasopharynx
d) Around margin for of bilateral choana
e) In right nasal cavity: attachment is along anterior half of superior turbinate
f) In left nasal cavity: attachment is along inferior surface of bulla.

Patient was advised with Dapsone 50mg PO OD for 3 months. Follow-up evaluation 3 wks later with nasopharyngolaryngoscopy demonstrated no local recurrence.

\section{Discussion}

The mode of treatment for rhinosporidosis has widely been anecdotal with little literature to support it [3]. Wide local surgical excision with electrocauterization of the base of the lesion is the treatment of choice [1-7,9,10]. Additionally, systematic dapsone may be prescribed for 1 year to prevent recurrence. Its mechanism is shown to arrest the maturation of sporangia and promote fibrosis in stroma [1-7,9]. There is a greater paucity of literature for the treatment of recurrent disease [3]. Surgery is effective at removing the lesion; however, resection with electrocautery can potentially cause localized tissue trauma that potentiates the possibility for inoculation $[1,5]$. Our patient had an uneventful surgical resection with the use of monopolar suction cautery. The Harmonic scalpel has been described in the literature to have superior hemostasis while preserving the integrity of local tissue $[3,11]$.

\section{Conclusion}

In this case report, the identification of recurrent rhinosporidiosis re-emphasized the importance of combined 
surgical and medical treatment to decrease local recurrence $[3,12,13]$. Incomplete removal \& lack of base cauterization is the major cause of recurrence of rhinosporidiosis. Dapsone has been shown to reduce the rate of recurrences. However its mechanism of action is unknown.

\section{References}

1. Arseculeratne SN (2002) Recent advances in rhinosporidiosis and Rhinosporidium seeberi. Indian J Med Microbiol 20(3): 119-131.

2. Ayub-ur-Rehman, Muhammad MN, Moallam FA Rhinosporidiosis. J Coll Physicians Surg Pak 22(10): 671-672.

3. Josue Chery, Christopher Bacskai, Ernesto Mendoza (2014) Recurrent Rhinosporidiosis. J of medical cases 5(2): 58-61.

4. Sonkhya N, Singhal P, Mishra P (2005) Naso-oropharyngeal rhinosporidiosis: endoscopic removal. Indian J Otolaryngol Head Neck Surg 57(4): 354-356.

5. Uledi S, Fauzia A (2011) Human nasal rhinosporidiosis: a case report from Malawi. Pan Afr Med J 9: 27.

6. Rajeshwari A, Gangadhaia S, Deviprasad S, Manohar S (2015) Rhinosporisiosis - a report of two cases. Indian J Otolaryngol Head Neck Surg 62(3): 322-325.
7. Puri R, Berry S, Mandal AK, Berry N (2000) Isolated trachael rhinosporidiosis - a case report. Indian J Otolaryngol Head Neck Surg 52(4): 380-381.

8. Mathew J, Padhy S, Lata S, Balchander H, Gopalakrishnan S (2010) Telelaryngoscopy-guided flexible fiberoptic intubation for laryngeal rhinosporidiosis. Int Anesthesia Res Soc 110(4): 1066-1068.

9. Das S, Kashyap B, Barua M, Gupta N, Saha R, et al. (2011) Nasal rhinosporidiosis in humans: new interpretations and a review of the literature of this enigmatic disease. Med Mycol 49(3): 311-315.

10. Ali A, Flieder D, Guiter G, Hoda SA (2001) Rhinosporidiosis: an unusual affliction. Arch Pathol Lab Med 125(10): 1392-1393.

11. PK Chatterjee, CR Khatua, SN Chaterjee, N Dastider (2013) Recurrent multiple Rhinosporidiosis with osteolytic lesion in hand \& foot a case report. The Journal of Laryngology \& Otology 91(8): 729-734.

12. Collison PJ, Weiner R (2004) Harmonic scalpel versus conventional tonsillectomy: a double-blind clinical trial. Ear Nose Throat J 83(10): 707-710.

13. Luca Morelli, Mario Polce, Francesco Piscioli, Franca Del Nonno, Renato Covello (2006) Human nasal rhinosporidiosis: an Italian case report. Diagn Pathol 1: 25.

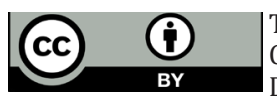

This work is licensed under Creative Commons Attribution 4.0 License DOI: $10.19080 /$ GJO.2017.09.555774 